\title{
THE HADAMARD CONJECTURE AND CIRCUITS OF LENGTH FOUR IN A COMPLETE BIPARTITE GRAPH
}

\author{
CHARLES H. C. LTTLE and DAVID J. THUENTE
}

(Received 26 August 1980)

Communicated by W. D. Wallis

\begin{abstract}
We show that the problem of settling the existence of an $n \times n$ Hadamard matrix, where $n$ is divisible by 4 , is equivalent to that of finding the cardinality of a smallest set $T$ of 4-circuits in the complete bipartite graph $K_{n, n}$ such that $T$ contains at least one circuit of each copy of $K_{2,3}$ in $K_{n, n}$. 1980 Mathematics subject classification (Amer. Math. Soc.): 05 C 50.

An Hadamard matrix is an $n \times n(1,-1)$-matrix in which the rows are mutually orthogonal. The Hadamard conjecture asserts that there exists an Hadamard matrix of order $n$ whenever $n$ is divisible by 4. (See Wallis (1972) and the references found therein.) In Little and Thuente (1979), we restate the conjecture as a problem concerning the 1-factors of a complete bipartite graph. In the present paper, the conjecture is shown to be equivalent to one about the circuits of length 4 in a complete bipartite graph.

We begin with a lemma.
\end{abstract}

Lemma 1. Let $S$ be a set with $|S|=n$ for some $n$ divisible by 4 . Suppose there exist subsets $T_{1}, T_{2}, \ldots, T_{n-1}$ of $S$, of cardinality $n / 2$, such that $\left|T_{i} \cap T_{j}\right|=n / 4$ whenever $i \neq j$. Then there exists an Hadamard matrix of order $n$.

Proof. Let $S=\left\{s_{1}, s_{2}, \ldots, s_{n}\right\}$. Define $H=\left(h_{i j}\right)$, where $h_{1 j}=1$ for all $j \in\{1,2, \ldots, n\}$ and, for all $i \in\{2,3, \ldots, n\}$,

$$
h_{i j}= \begin{cases}1 & \text { if } s_{j} \in T_{i-1} \\ -1 & \text { otherwise. }\end{cases}
$$

CC Copyright Australian Mathematical Society 1981 
Since $\left|T_{i}\right|=n / 2$ for all $i$, the first row is orthogonal to all the others. Furthermore since $\left|T_{i}\right|=n / 2,\left|T_{j}\right|=n / 2$ and $\left|T_{i} \cap T_{j}\right|=n / 4$ for all $j \neq i$, we must have $\left|T_{j}-T_{i}\right|=\left|T_{i}-T_{j}\right|=n / 4$, so that $\left|\bar{T}_{i} \cap \bar{T}_{j}\right|=n / 4$ where $\bar{T}_{i}=S-T_{i}$ and $\bar{T}_{j}=S-T_{j}$. It follows that rows $i+1$ and $j+1$ are orthogonal. Hence $H$ is an Hadamard matrix of order $n$.

As an example, let $S=\left\{s_{1}, s_{2}, s_{3}, s_{4}\right\}, T_{1}=\left\{s_{1}, s_{2}\right\}, T_{2}=\left\{s_{1}, s_{3}\right\}$, and $T_{3}=$ $\left\{s_{1}, s_{4}\right\}$. Then

$$
H=\left(\begin{array}{rrrr}
1 & 1 & 1 & 1 \\
1 & 1 & -1 & -1 \\
1 & -1 & 1 & -1 \\
1 & -1 & -1 & 1
\end{array}\right)
$$

The equivalence of the Hadamard conjecture with a problem on the 4-circuits of a complete bipartite graph is shown in the following theorem.

THEOREM. Let $S$ be the set of all 4-circuits of $K_{n, n}$ where $n$ is even. Let $S_{1}, \ldots, S_{k}$ be the collection of all subsets $S_{i}$ of $S$, of cardinality 3 , such that the union of the three circuits of $S_{i}$ is $K_{2,3}$. Let $T$ be a smallest subset of $S$ such that $T \cap S_{i} \neq \varnothing$ for each $i$. Then $|T| \geqslant \frac{1}{8} n^{2}(n-1)(n-2)$, and equality holds if and only if there exists an Hadamard matrix of order $n$.

Proof. Let $A$ be an $n \times n(1,-1)$-matrix $\left(a_{i j}\right)$. Let $K_{n, n}$ be the complete bipartite graph with vertex set $\left\{v_{1}, v_{2}, \ldots, v_{n}, w_{1}, w_{2}, \ldots, w_{n}\right\}$, where $v_{i}$ and $w_{j}$ are adjacent for each $i$ and $j$. Furthermore, for each $i$ and $j$ let the edge joining $v_{i}$ to $w_{j}$ be directed from $v_{i}$ to $w_{j}$ if $a_{i j}=1$ and from $w_{j}$ to $v_{i}$ otherwise.

Note that a pair of rows and a pair of columns of $A$ corresponds in an obvious way to an undirected 4-circuit in $K_{n, n}$. We say that this 4-circuit is clockwise even if the number of edges directed in the clockwise sense is even, and clockwise odd otherwise. Let $C$ be a 4-circuit of $K_{n, n}$ with vertex set $\left\{v_{h}, v_{i}, w_{j}, w_{k}\right\}$. If $a_{h j}=a_{i j}$, then exactly one of the two edges of $C$ incident on $w_{j}$ is directed in the clockwise sense. If $a_{h j} \neq a_{i j}$, then those edges are directed in the same sense on $C$. Analogous results hold for $a_{h k}$ and $a_{i k}$. It follows that $C$ is clockwise odd if and only if exactly one of the equations $a_{h j}=a_{i j}$ and $a_{h k}=a_{i k}$ holds. This condition holds if and only if $a_{h j} a_{i j}+a_{h k} a_{i k}=0$.

If we let $X_{h i}$ be the set of columns $j$ of $A$ for which $a_{h j}=a_{i j}$ and let $Y_{h i}$ be the set of all the remaining columns of $A$, it follows from the above considerations that the number of clockwise odd 4-circuits containing $v_{h}$ and $v_{i}$ is $\left|X_{h i}\right|\left|Y_{h i}\right|$. This product is a maximum if $\left|X_{h i}\right|=\left|Y_{h i}\right|$, and this condition holds if and only if rows $h$ and $i$ of $A$ are orthogonal. It follows that the number of clockwise odd 4-circuits of $K_{n, n}$ is maximised if $A$ is an Hadamard matrix. 
Suppose therefore that $A$ is an Hadamard matrix. Then $\left|X_{k i}\right|=\left|Y_{h i}\right|=\frac{1}{2} n$ for all $h$ and $i$, so that there are $\left(\frac{1}{2} n\right)^{2}$ clockwise odd 4-circuits of $K_{n, n}$ containing $v_{h}$ and $v_{i}$. Therefore $K_{n, n}$ has $\frac{1}{4}\left(\begin{array}{l}n \\ 2\end{array}\right) n^{2}$ clockwise odd 4-circuits altogether, and therefore $\left(\begin{array}{l}n \\ 2\end{array}\right)^{2}-\frac{1}{4}\left(\begin{array}{l}n \\ 2\end{array}\right) n^{2}=\frac{1}{8} n^{2}(n-1)(n-2)$ clockwise even ones. Let $T_{0}$ be the set of all clockwise even 4-circuits of $K_{n, n}$.

The graph $K_{2,3}$ is drawn in Figure 1, where an orientation is given in which all three circuits are clockwise even. Since every edge of $K_{2,3}$ belongs to exactly two circuits of $K_{2,3}$, it follows that for any orientation of $K_{2,3}$ there are an odd number of clockwise even circuits. It follows that $T_{0} \cap S_{i} \neq \varnothing$ for all $i$.

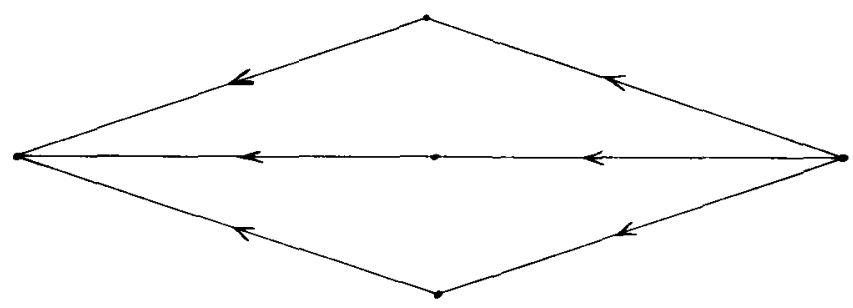

Figure 1

We have now proved that if there exists an Hadamard matrix of order $n$, then $|T|<\frac{1}{8} n^{2}(n-1)(n-2)$. We prove next that in fact $|T|>\frac{1}{8} n^{2}(n-1)(n-2)$. The existence of an $n \times n$ Hadamard matrix will then imply that $|T|=$ $\frac{1}{8} n^{2}(n-1)(n-2)$. We will then prove the converse.

Suppose therefore that $T \cap S_{i} \neq \varnothing$ for all $i$. We consider first those copies of $K_{2,3}$ in $K_{n, n}$ which contain exactly three vertices of $\left\{v_{1}, \ldots, v_{n}\right\}$. We denote the complement of $K_{n, n}$ by $2 K_{n}$, since it has exactly two components, $C_{1}$ and $C_{2}$, each isomorphic to $K_{n}$. Let $C_{1}$ be the component with vertex set $\left\{v_{1}, \ldots, v_{n}\right\}$. Then the complement (in $K_{5}$ ) of a copy of $K_{2,3}$ containing three vertices of $\left\{v_{1}, \ldots, v_{n}\right\}$ is $P_{1} \cup P_{2}$, where $P_{1}$ is a triangle of $C_{1}$ and $P_{2}$ an edge of $C_{2}$. The complement (in $K_{4}$ ) of a circuit in $K_{2,3}$ is then the union of $P_{2}$ with an edge of $P_{1}$. Let us now temporarily fix $P_{2}$ and let $P_{1}$ run through all triangles in $C_{1}$. In order to contain at least one circuit in each of the corresponding copies of $K_{2,3}$, $T$ must contain at least as many circuits as the cardinality of the smallest set of edges whose deletion from $K_{n}$ yields a graph with no triangles, and furthermore each such circuit must contain both end-vertices of the edge $\boldsymbol{P}_{\mathbf{2}}$. By a well known theorem of Turán (see Turán (1941) or Harary (1969) p. 17), the largest subgraph of $K_{n}$ having no triangles is $K_{n / 2, n / 2}$, since $n$ is even. Since $K_{n}$ has $\left(\begin{array}{l}n \\ 2\end{array}\right)$ edges and $K_{n / 2, n / 2}$ has $\frac{1}{4} n^{2}$ edges, $T$ must contain at least $\left(\begin{array}{c}n \\ 2\end{array}\right)-\frac{1}{4} n^{2}$ circuits which include the end-vertices of $P_{2}$. Since there are $\left(\begin{array}{l}n \\ 2\end{array}\right)$ choices for $P_{2}$, it follows that $|T| \geqslant\left(\begin{array}{l}n \\ 2\end{array}\right)\left[\left(\begin{array}{c}n \\ 2\end{array}\right)-\frac{1}{4} n^{2}\right]=\frac{1}{8} n^{2}(n-1)(n-2)$. 
We continue the argument under the assumption that

$$
|T|=\frac{1}{8} n^{2}(n-1)(n-2)
$$

and prove the existence of an $n \times n$ Hadamard matrix. We now consider the copies of $K_{2,3}$ in $K_{n, n}$ which have only two vertices of $\left\{v_{1}, \ldots, v_{n}\right\}$. The complement (in $K_{5}$ ) of such a copy of $K_{2,3}$ is $P_{1} \cup P_{2}$ where $P_{1}$ is an edge of $C_{1}$ and $P_{2}$ a triangle of $C_{2}$. The complement (in $K_{4}$ ) of any circuit in such a copy $Z$ of $K_{2,3}$ is the union of $P_{1}$ with an edge $e$ of $P_{2}$. We have already seen that in order to include at least one circuit of each copy of $K_{2,3}$ that includes the end-vertices of $e$ and three vertices of $\left\{v_{1}, \ldots, v_{n}\right\}, T$ must contain all the 4-circuits whose complements in $K_{4}$ are pairs of edges where one edge of the pair is $e$ and the other is chosen from the complement, $2 K_{n / 2}$, in $C_{1}$ of a fixed copy of $K_{n / 2, n / 2}$. In order to ensure that $T$ contains a circuit of $Z$, the copies of $K_{n / 2, n / 2}$ in $C_{1}$ corresponding to the edges of $P_{2}$ must be chosen in such a way that the edge $P_{1}$ appears in the complement of at least one of them. Since $P_{1}$ is any edge of $C_{1}$, we find that $C_{1}$ must be the union of three copies of $2 K_{n / 2}$, each copy being the complement in $C_{1}$ of a copy of $K_{n / 2, n / 2}$ chosen to correspond to an edge of $P_{2}$. Since $P_{2}$ is any triangle of $C_{2}$, we see that to each edge of $C_{2}$ there corresponds a subgraph $2 K_{n / 2}$ of $C_{1}$ in such a way that for any triangle of $C_{2}$ the union of the corresponding subgraphs of $C_{1}$ is $C_{1}$ itself. For any edge $e$ of $C_{2}$, let us denote by $V_{1}(e)$ and $V_{2}(e)$ the vertex sets of the copies of $K_{n / 2}$ in the subgraph $2 K_{n / 2}$ of $C_{1}$ corresponding to $e$. Thus $\left|V_{1}(e)\right|=\left|V_{2}(e)\right|=\frac{1}{2} n$ for each e.

Let us now consider a triangle of $C_{2}$ with edge set $\left\{e_{1}, e_{2}, e_{3}\right\}$. Since $C_{1}$ is the union of the corresponding copies of $2 K_{n / 2}$, each pair of vertices of $C_{1}$ must be contained in at least one of the sets $V_{i}\left(e_{j}\right)$ where $e \in\{1,2\}$ and $j \in\{1,2,3\}$. It follows that

$$
\begin{aligned}
\left\{V_{1}\left(e_{3}\right), V_{2}\left(e_{3}\right)\right\}=\{[ & \left.V_{1}\left(e_{1}\right) \cap V_{1}\left(e_{2}\right)\right] \cup\left[V_{2}\left(e_{1}\right) \cap V_{2}\left(e_{2}\right)\right], \\
& {\left.\left[V_{1}\left(e_{1}\right) \cap V_{2}\left(e_{2}\right)\right] \cup\left[V_{2}\left(e_{1}\right) \cap V_{1}\left(e_{2}\right)\right]\right\} . }
\end{aligned}
$$

Note that $\left|V_{1}\left(e_{1}\right) \cap V_{1}\left(e_{2}\right)\right|=\left|V_{2}\left(e_{1}\right) \cap V_{2}\left(e_{2}\right)\right|$, since

$$
\left|V_{1}\left(e_{1}\right)\right|=\left|V_{2}\left(e_{2}\right)\right|,\left|V_{1}\left(e_{1}\right)\right|=\left|V_{1}\left(e_{1}\right) \cap V_{1}\left(e_{2}\right)\right|+\left|V_{1}\left(e_{1}\right) \cap V_{2}\left(e_{2}\right)\right|
$$

and $\left|V_{2}\left(e_{2}\right)\right|=\left|V_{1}\left(e_{1}\right) \cap V_{2}\left(e_{2}\right)\right|+\left|V_{2}\left(e_{1}\right) \cap V_{2}\left(e_{2}\right)\right|$. Since

$$
\left|V_{1}\left(e_{1}\right) \cap V_{1}\left(e_{2}\right)\right|+\left|V_{2}\left(e_{1}\right) \cap V_{2}\left(e_{2}\right)\right|=\left|V_{1}\left(e_{3}\right)\right|=\left|V_{2}\left(e_{3}\right)\right|=\frac{1}{2} n,
$$

it follows that $n$ is divisible by 4 and $\left|V_{1}\left(e_{1}\right) \cap V_{1}\left(e_{2}\right)\right|=\frac{1}{4} n$.

Finally we consider a subgraph $K_{1, n-1}$ of $C_{2}$. Any pair of the $n-1$ edges $f_{1}, \ldots, f_{n-1}$ in this subgraph form two sides of a triangle in $C_{2}$. It is now immediate that the sets $V_{1}\left(f_{1}\right), \ldots, V_{1}\left(f_{n-1}\right)$ satisfy the conditions of Lemma 1. The existence of an Hadamard matrix of order $n$ follows. 


\section{References}

F. Harary (1969), Graph theory (Addison-Wesley, London).

C. H. C. Little and D. J. Thuente (1979), 'Complete bipartite graphs and the Hadamard conjecture', Proc. Tenth Southeastern Conference on Combinatorics, Graph Theory and Computing, Florida Atlantic Univ., Boca Raton, edited by F. Hoffman, D. McCarthy, R. C. Mullin and R. G. Stanton, pp. 721-726. (Utilitas Mathematica, Winnipeg).

P. Turán (1941), 'Eine Extremalaufgabe aus der Graphentheorie', Mat. Fiz. Lapok 48, 436-452.

J. S. Wallis (1972), Hadamard matrices, Lecture Notes in Mathematics 292, 273-489 (SpringerVerlag, New York).

Department of Mathematics

Royal Melbourne Institute of Technology Ltd.

Melbourne, Victoria 3000

Australia
Department of Mathematics

Purdue University at Fort Wayne

Indiana 46805

U.S.A. 